Annales Academiæ Scientiarum Fennicæ

Mathematica

Volumen 38, 2013, 565-580

\title{
CARATHÉODORY AND SMIRNOV TYPE THEOREMS FOR HARMONIC MAPPINGS OF THE UNIT DISK ONTO SURFACES
}

\author{
David Kalaj, Marijan Marković and Miodrag Mateljević \\ University of Montenegro, Faculty of Natural Sciences and Mathematics \\ Cetinjski put b.b., 81000 Podgorica, Montenegro; davidk@ac.me \\ University of Montenegro, Faculty of Natural Sciences and Mathematics \\ Cetinjski put b.b., 81000 Podgorica, Montenegro; marijanmmarkovic@gmail.com \\ University of Belgrade, Faculty of Mathematics \\ Studentski trg 16, 11000 Belgrade, Serbia; miodrag@matf.bg.ac.rs
}

\begin{abstract}
We prove representation theorems, the versions of Smirnov's theorem and Carathéodory type theorem for harmonic homeomorphisms of the unit disk onto Jordan surfaces with rectifiable boundaries. Further we establish the classical isoperimetric inequality and the Riesz-Zygmund inequality for Jordan harmonic surfaces without any smoothness assumptions on the boundary.
\end{abstract}

\section{Introduction}

By $\langle\cdot, \cdot\rangle$ and $|\cdot|$ are denoted the standard inner product and Euclidean norm in the space $\mathbf{R}^{n}$. In particular $\mathbf{C}^{n}=\mathbf{R}^{2 n}$, where $\mathbf{C}=\mathbf{R}^{2}$ is the complex plane. By $\mathbf{U}=\{z=x+i y \in \mathbf{C}:|z|<1\}$ we denote the unit disk and by $\mathbf{T}=\{\zeta \in \mathbf{C}:|\zeta|=1\}$ is denoted the unit circle in the complex plane. It is also convenient to write complex numbers in the polar form $r e^{i t}$ (where $r \geq 0$ and $t \in \mathbf{R}$ ).

Let $f=\left(f^{1}, f^{2}, \ldots, f^{n}\right): \mathbf{U} \rightarrow \mathbf{R}^{n}$ be a mapping defined in the unit disc having the partial derivatives of the first order in $\mathbf{U}$. The formal derivative (Jacobian matrix) of $f$ is defined by

Denote

$$
\nabla f=\left(f_{x}, f_{y}\right)=\left(\begin{array}{cc}
f_{x}^{1} & f_{y}^{1} \\
\vdots & \vdots \\
f_{x}^{n} & f_{y}^{n}
\end{array}\right)
$$

$$
J_{f}=\left(\operatorname{det}(\nabla f)^{t}(\nabla f)\right)^{1 / 2}=\sqrt{\left|f_{x}\right|^{2}\left|f_{y}\right|^{2}-\left\langle f_{x}, f_{y}\right\rangle^{2}} .
$$

A mapping $f=\left(f^{1}, f^{2}, \ldots, f^{n}\right): \mathbf{U} \rightarrow \mathbf{R}^{n}$ is called harmonic if each $f^{j}(j=$ $1,2, \ldots, n)$ is (real-valued) harmonic functions in $\mathbf{U}$, that is if $f^{j}$ is twice differentiable and satisfies the well known Laplace equation $\Delta f^{j} \equiv 0$. Let

$$
P(r, t)=\frac{1-r^{2}}{2 \pi\left(1-2 r \cos t+r^{2}\right)}, \quad 0 \leq r<1,0 \leq t \leq 2 \pi,
$$

denote the Poisson kernel for the disc U. It is known that every bounded harmonic mapping $f: \mathbf{U} \rightarrow \mathbf{R}^{n}$ has the representation as the Poisson integral

$$
f(z)=\mathrm{P}[\phi](z)=\int_{0}^{2 \pi} P(r, t-\theta) \phi(t) d t, \quad z=r e^{i \theta} \in \mathbf{U},
$$

doi:10.5186/aasfm.2013.3822

2010 Mathematics Subject Classification: Primary 30C55; Secondary 31C05.

Key words: Harmonic mappings, harmonic surfaces, isoperimetric inequality. 
where $\phi:[0,2 \pi] \rightarrow \mathbf{R}^{n}, \phi(0)=\phi(2 \pi)$ is a measurable and bounded in the segment $[0,2 \pi]$ (i.e., $\left.\phi \in L^{\infty}[0,2 \pi]\right)$.

A homeomorphic image (in the literature it is also said that a mapping $\Gamma$ ) of the unit circle $\mathbf{T}$ in $\mathbf{R}^{n}$ is called a closed Jordan curve. Here it is convenient to identify the mapping $\Gamma$ with the trace $\Gamma(\mathbf{T})$. In this paper, we will be mainly concerned with the case that $\Gamma$ is rectifiable, we denote by $|\Gamma|$ its length, and with closed Jordan harmonic surfaces with rectifiable boundaries. We will precise in the sequel the last notation. Examples 1.1 and 1.2 show that it is a reasonable sufficient condition for our purposes.

A closed Jordan surface $\Sigma \subseteq \mathbf{R}^{n}$ is a homeomorphic image of the closed unit disk, i.e., $\Sigma=\Phi(\overline{\mathbf{U}})$, where $\Phi$ is a homeomorphism. We say that $\Sigma$ is spanned by the Jordan curve $\Gamma=\partial \Sigma=\Phi(\mathbf{T})$. If $\Gamma$ is a rectifiable curve, we say that the surface $\Sigma$ is a closed Jordan surface with rectifiable boundary and also call $\Sigma_{o}=\Sigma \backslash \partial \Sigma$ an (open) Jordan surface with rectifiable boundary. Just a homeomorphic image of the unit disc we call simply a open Jordan surface.

A open Jordan surface $\Sigma_{o} \subseteq \mathbf{R}^{n}$ is regular if $\Sigma_{o}=\tau(\mathbf{U})$, where $\tau=\tau(x, y)$ is an injective mapping of class $C^{1}$ with positive Jacobian $J_{\tau}$ in $\mathbf{U}$. Thus the tangent vectors $\tau_{x}, \tau_{y}$ are linearly independent for all $z=x+i y \in \mathbf{U}$ or equivalently the Jacobian matrix $\nabla \tau$ has full rank 2 in the whole domain $\mathbf{U}$. The mapping $\tau$ is called a parametrization of $\Sigma_{o}$. Certainly, it is not unique. The area $\left|\Sigma_{o}\right|$ of the surface $\Sigma_{o}$ equals

$$
\left|\Sigma_{o}\right|=\int_{\mathbf{U}} J_{\tau}(z) d A(z)
$$

where $d A(z)=d x d y$ is the Lebesgue measure in the complex plane.

We call a open Jordan surface $\Sigma_{o} \subseteq \mathbf{R}^{n}$ a (simply-connected) harmonic surface if there exists a homeomorphic harmonic mapping $\tau: \mathbf{U} \stackrel{\text { onto }}{\longrightarrow} \Sigma_{o}$. We call a closed Jordan surface $\Sigma \subseteq \mathbf{R}^{n}$ a Jordan closed harmonic surface if there exists a homeomorphic harmonic mapping $\tau: \mathbf{U} \stackrel{\text { onto }}{\longrightarrow} \Sigma_{o}=\Sigma \backslash \Gamma$ (it need not have a homeomorphic extension to $\overline{\mathbf{U}}$, for a counterexample see [7] and Example 1.2). Let us point out that in general setting a parametrization $\tau$ of a (open) Jordan harmonic surface need not be a regular parametrization, i.e., the strict inequality

$$
J_{\tau}=\sqrt{\left|\tau_{x}\right|^{2}\left|\tau_{y}\right|^{2}-\left\langle\tau_{x}, \tau_{y}\right\rangle^{2}}>0 \text { in the whole disc } \mathbf{U}
$$

need not hold except in the planar case (in view of Lewy's theorem, see [13]). In other words, the harmonic surfaces in the sense of our definition may have branch points, i.e., the points with zero Jacobian. Notice also the following important fact. If a surface $\Sigma_{o} \subset \mathbf{R}^{n}$ is enough regular (for example $\Sigma_{o} \in C^{3}$ ) and $\tau$ is a harmonic homeomorphism of the unit disk onto $\Sigma_{o}$, then $\tau$ is a diffeomorphism (cf. [11, Theorem 9.3]).

Together with the introduction, the paper contains two more sections. In the second section it is proved that a harmonic homeomorphism of the unit disk onto a (open) Jordan surface with rectifiable boundary has BV-extension (of bounded variation) onto the boundary. This result is an extension of Hengartner and Schober theorem proved in [9], and presents a generalization of the classical Carathéodory theorem. In addition a generalization of the Smirnov theorem is proved for harmonic mappings of the unit disk onto a Jordan surface, see Theorem 2.7, which asserts 
that, the angular derivative of a harmonic homeomorphism $f$ belongs to the (vectorvalued) harmonic Hardy class

$$
h^{1}=\left\{f: \mathbf{U} \rightarrow \mathbf{R}^{n}: f \text { is harmonic and }\|f\|_{1}=\sup _{0<r<1} \int_{0}^{2 \pi}\left|f\left(r e^{i t}\right)\right| d t<\infty\right\}
$$

if and only if the boundary of the surface $f(\mathbf{U})$ is rectifiable. In the third section it is proved the isoperimetric inequality for harmonic surfaces. More precisely, in the classical notations, if $A$ is the area of a harmonic Jordan surface $\Sigma$ and $L$ is the length of its circumference, then there holds the inequality

$$
4 \pi A \leq L^{2} .
$$

The last result is not surprising, it can be found in the literature in various formulations. However we believe that our inequality contains some new information regarding the isoperimetric inequality, because it is proved under some optimal conditions of smoothness of the boundary. We finish the paper by establishing the Riesz-Zygmund inequality and related geometric results. These results imply that the perimeter of a Jordan closed harmonic surface is bigger than or equal to two "diameters". Examples 1.1 and 1.2 show that it is not true for harmonic surfaces in general. More precisely, we say that $D$ satisfies perimeter-diameter (Riesz-Zygmund) property if $\operatorname{diam}(D) \leq \frac{1}{2} H_{1}(\partial D)$. Here $H_{1}$ denotes the 1-dimensional Hausdorff measure (also called arc length measure). Recall that a set $E \subseteq C$ is said to be rectifiable if there exist a countable family of rectifiable curves $C_{k}$ such that $H_{1}\left(E \backslash \bigcup_{k} C_{k}\right)=0$. We will prove if $\phi:[0,2 \pi] \rightarrow \mathbf{R}^{n}$ is of bounded variation, $f=\mathrm{P}[\phi]$ and $D=f(\mathbf{U})$, then $\operatorname{diam}(D) \leq \frac{1}{2} V(\phi)$ (see Theorem 3.12 below). Note that we have also announced Theorem 2.7 and a version of the isoperimetric inequality for harmonic functions in [15].

Example 1.1. Define $f(w)=(c v, \Re(\cos w), \Re(\sin w)), w=u+i v, A=(0,2 \pi) \times$ $(-1,1)$, and $D=f(A)$. Then $D$ is a catenoid with cut along a curve and also a simple connected harmonic surface. This surface does not satisfy Riesz-Zygmund property for $c$ big enough (see Figure 1). $f$ is continuous on $\bar{A}$. The boundary of $\bar{D}$ consists of two circles.

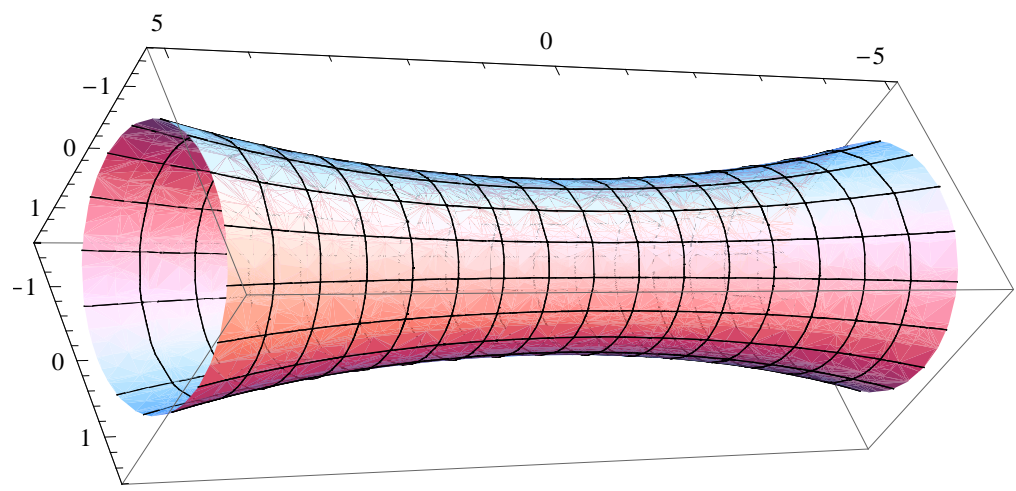

Figure 1. Catenoid for $c=5$. 
Example 1.2. (cf. [7]) Assume that $m>2$ is an integer and $0=\theta_{0}<\theta_{1}<$ $\cdots<\theta_{m}<\theta_{m+1}=2 \pi$ and define

$$
\varphi=\sum_{j=0}^{m} \theta_{j} \chi_{\left[\theta_{j}, \theta_{j+1}\right]}
$$

Then $f=\mathrm{P}[\phi]$, where $\phi(t)=e^{i \varphi(t)}, t \in[0,2 \pi]$, is a harmonic diffeomorphism of the unit disk onto a Jordan domain enclosed by the polygonal line with vertices $e^{i \theta_{j}}, j=0,1, \ldots, m$. It can be easily modified into a map of the unit disk onto a harmonic surface $\Sigma$ as follows. Define

$$
F(z)=(h(z), \Re(f(z)), \Im(f(z))),
$$

where $h$ is an arbitrary real harmonic function continuous up to the boundary. Then the cluster set of $F$ at a point $e^{i \theta_{j}}$ is the segment

$$
\left[\left(h\left(e^{i \theta_{j}}\right), \cos \theta_{j}, \sin \theta_{j}\right),\left(h\left(e^{i \theta_{j}}\right), \cos \theta_{j+1}, \sin \theta_{j+1}\right)\right] .
$$

Take for example

$$
\varphi(t)= \begin{cases}\frac{-2 \pi}{3}, & -\pi \leq t \leq-\frac{\pi}{3} \\ 0, & -\frac{\pi}{3}<t \leq \frac{\pi}{3} \\ \frac{2 \pi}{3}, & \frac{\pi}{3}<t \leq \pi\end{cases}
$$

Then for $\phi(t)=e^{i \varphi(t)}, t \in[0,2 \pi]$, we have

$$
f(z)=\mathrm{P}[\phi](z)=\frac{1}{\pi} \sum_{k=0}^{2} \beta^{2 k} \arg \left(\frac{z-\beta^{2 k+1}}{z-\beta^{2 k-1}}\right), \quad \beta=e^{i \pi / 3} .
$$

a) Define $F(z)=(5 \Re z, \Re f(z), \Im f(z))$. Then $F$ defines the harmonic surface $\Sigma=F(\mathbf{U}) \subseteq \mathbf{R}^{3}$ in the Figure 2. Its boundary consists of six segments which do not make a polygon (the upper edge is a part of the boundary) and it is homeomorphic to the set $\mathbf{T} \cup[0,1]$, which is the union of the unit circle and the segment $[0,1]$. Note that $\Sigma$ is a Jordan surface with a cut along a segment.

b) If instead of $F$ we take $\tilde{F}=\left(\Re f(z), \Im f(z), \frac{1}{\pi} \arg (1+z)\right)$, then we obtain a harmonic Jordan surface (Figure 3). Its boundary consists of six segments which make a polygon.

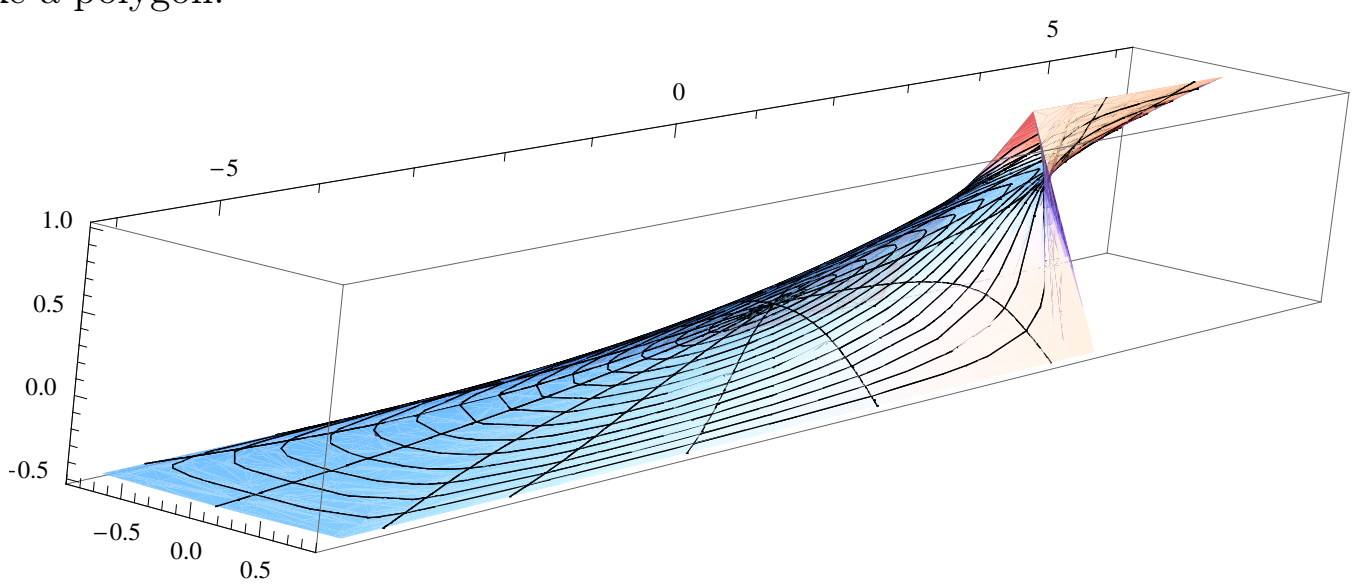

Figure 2. A harmonic surface which coincides with a Jordan surface with a cut along the upper edge (it is not a Jordan surface). 


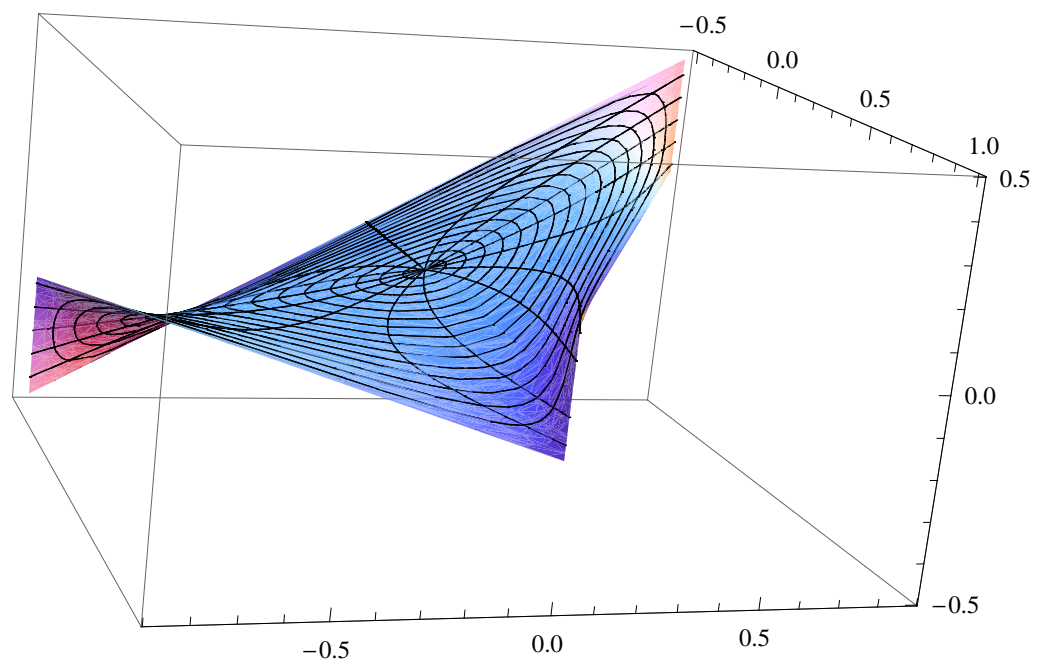

Figure 3. A harmonic Jordan surface.

\section{Carathéodory and Smirnov theorem for harmonic mappings}

Recall that a vector-valued function $\phi$ on the real line is said to be of bounded variation on a chosen interval $[a, b]$ if its total variation $V(\phi)=V_{a}^{b}(\phi)$ is finite, i.e., $\phi \in \mathrm{BV}([a, b])$ if and only if $V_{a}^{b}(\phi)<\infty$. The graph of a function having this property is well behaved in a precise sense. A characterization states that the real-valued functions of bounded variation on a closed interval are exactly those $\phi$ which can be written as a difference $\phi_{1}-\phi_{2}$, where both $\phi_{1}$ and $\phi_{2}$ are bounded and monotone. This result is known as the Jordan decomposition. Moreover, if $\phi$ is absolutely continuous on $[a, b]$, then $V_{a}^{b}(\phi)=\int_{a}^{b}\left|\phi^{\prime}(t)\right| d t$.

Lemma 2.1. (Helly selection theorem, see [17]) Let $\left(\phi_{n}\right)$ be a sequence of uniformly bounded functions of uniformly bounded variation on a segment $[a, b]$. Then there exists a subsequence $\left(\phi_{n_{k}}\right) \subseteq\left(\phi_{n}\right)$ such that $\phi_{n_{k}}(x) \rightarrow \phi(x)$ as $k \rightarrow \infty$ for every $x \in[a, b]$ and $\phi$ is of bounded variation. Moreover if each $\phi_{n}$ is monotone increasing (or decreasing), then so is $\phi$.

The following theorem yields a useful representation of harmonic homeomorphisms by means of functions of bounded variations.

Theorem 2.2. Let $\Sigma \subseteq \mathbf{R}^{n}$ be a closed Jordan surface with rectifiable boundary $\Gamma, \Sigma_{o}=\Sigma \backslash \Gamma$ and $f: \mathbf{U} \rightarrow \Sigma_{o}$ be a harmonic homeomorphism of the unit disk onto $\Sigma_{o}$. Then there exists a function $\phi:[0,2 \pi] \rightarrow \Gamma, \phi(0)=\phi(2 \pi)$ with bounded variation and with at most a countable set of points of discontinuity, where it has the left and the right limit, such that $f=\mathrm{P}[\phi]$.

Proof. Let $\Phi: \overline{\mathbf{U}} \rightarrow \Sigma$ be a homeomorphism onto the Jordan surface $\Sigma$. The function $F=\Phi^{-1} \circ f: \mathbf{U} \rightarrow \mathbf{U}$ is also a homomorphism and $|\Phi|_{\infty}=\max \{|\Phi(z)|: z \in$ $\overline{\mathbf{U}}\}$. Let $s_{n}=\frac{n-1}{n}$, where $n \geq 2$ is an integer, $U_{n}=\left\{z:|z|<s_{n}\right\}$ and $\Delta_{n}=F^{-1}\left(U_{n}\right)$ and let $g_{n}$ be a conformal mapping of $\mathbf{U}$ onto the domain $\Delta_{n}$ such that $g_{n}(0)=0$ and $g_{n}^{\prime}(0)>0$. We can assume w.l.g. that $0 \in \Delta_{n}$ (for all $\left.n\right)$. Then the function

$$
F_{n}=\frac{n}{n-1}\left(F \circ g_{n}\right)=\frac{n}{n-1}\left(\Phi^{-1} \circ f \circ g_{n}\right): \overline{\mathbf{U}} \rightarrow \overline{\mathbf{U}}
$$


is a homeomorphism. Let $\varphi_{n}=\left.F_{n}\right|_{\mathbf{T}}$. Then $\varphi_{n}\left(e^{i \theta}\right)=e^{i \phi_{n}(\theta)}$ such that $\phi_{n}(\theta)$ is a monotone function and $\left.\left(f \circ g_{n}\right)\right|_{\mathbf{T}}=\Phi \circ\left(s_{n} \varphi_{n}\right)$, where we recall that $s_{n}=\frac{n-1}{n}$. By Theorem 2.1 there exists a convergent subsequence $\left(\phi_{n_{k}}\right)$ of $\left(\phi_{n}\right)$. Let $\phi_{0}=\lim \phi_{n_{k}}$ and $\varphi_{0}=\lim \varphi_{n_{k}}$. Then $\phi_{0}$ is monotone and of bounded variation. Therefore

$$
\left.\frac{n_{k}}{n_{k}-1}\left(\Phi^{-1} \circ f \circ g_{n_{k}}\right)\right|_{\mathbf{T}} \rightarrow \varphi_{0} .
$$

It follows that

$$
\lim _{k \rightarrow \infty}\left(f \circ g_{n_{k}}\right)\left(e^{i \theta}\right)=\Phi\left(\varphi_{0}\left(e^{i \theta}\right)\right) \text { for every } \theta
$$

since $\Phi^{-1}$ is a homeomorphism $\Sigma$ onto $\overline{\mathbf{U}}$. Since $\Gamma$ is a rectifiable curve by Scheeffer's theorem (see [21]), the function $\Phi$ is of bounded variation on $\mathbf{T}$. Hence, since $\phi_{0}$ is monotone and of bounded variation, it follows that the mapping $\phi\left(e^{i \theta}\right)=\Phi\left(\varphi_{0}\left(e^{i \theta}\right)\right.$ is also of bounded variation.

Functions $f_{k}=\left.\left(f \circ g_{n_{k}}\right)\right|_{\mathbf{T}}$ are continuous uniformly bounded by $|\Phi|_{\infty}$ and $f \circ g_{n_{k}}$ are harmonic. According to the Lebesgue Dominated Convergence Theorem, for $z \in \mathbf{U}$ we obtain

$$
\lim _{k \rightarrow \infty}\left(f \circ g_{n_{k}}\right)(z)=\lim _{k \rightarrow \infty} \mathrm{P}\left[f_{k}\right](z)=\mathrm{P}\left[\Phi \circ \varphi_{0}\right](z) .
$$

It follows that the sequence $g_{n_{k}}$ converges. Let $g_{0}(z)=\lim _{k \rightarrow \infty} g_{n_{k}}(z)$. Since $g_{0}$ is a conformal mapping of the unit disk onto itself such that $g_{0}(0)=0$ and $g_{0}^{\prime}(0)>0$, it follows that $g_{0}=\mathrm{Id}$. Therefore $f=\mathrm{P}[\phi]$, where $\phi=\Phi \circ \varphi_{0}$. Finally, using that $\Phi$ is continuous and $\varphi_{0}$ is monotone, we conclude that the mapping $\phi$ is continuous except in a countable set of points where it has the left and the right limit.

The following proposition is known (see e.g. [7, Section 1.4]).

Proposition 2.3. Suppose that $f=\mathrm{P}[\phi]$, where $\phi(0)=\phi(2 \pi), \phi \in L^{\infty}[0,2 \pi]$, and that for some $\theta_{0} \in[0,2 \pi]$ there holds

$$
\lim _{\theta \uparrow \theta_{0}} \phi(\theta)=A_{0}
$$

and

$$
\lim _{\theta \downarrow \theta_{0}} \phi(\theta)=B_{0} .
$$

Let for $\lambda \in[-1,1], \Gamma_{\lambda}(s) \subseteq \mathbf{U}, 0 \leq s<1$ be a linear segment emanating at $\Gamma_{\lambda}(1)=e^{i \theta_{0}}$ and forming the angle $-\frac{\pi \lambda}{2}$ with $e^{i \theta_{0}}$. Then we have

$$
\lim _{s \rightarrow 1^{-}} f\left(\Gamma_{\lambda}(s)\right)=\frac{1}{2}(1-\lambda) A_{0}+\frac{1}{2}(1+\lambda) B_{0} .
$$

Of course, if $A_{0}=B_{0}=\phi\left(\theta_{0}\right)$ (that is if $\phi$ is continuous at $\theta_{0} \in[0,2 \pi]$ ), then it is well known that $f$ has a continuous extension on $e^{i \theta_{0}} \cup \mathbf{U}$.

Let $f$ be defined in $\mathbf{U}$. At any point $\zeta \in \mathbf{T}$, the cluster set $C_{\mathbf{U}}(f, \zeta)$ is defined as follows: $\alpha \in C_{\mathbf{U}}(f, \zeta)$ if there exists a sequence $\left(z_{n}\right) \subseteq \mathbf{U}$ such that $\lim _{n \rightarrow \infty} z_{n}=\zeta$ while $\lim _{n \rightarrow \infty} f\left(z_{n}\right)=\alpha$. It is known that for any $\zeta$ the cluster set $C_{\mathbf{U}}(f, \zeta)$ is nonempty and closed.

Theorem 2.4. Let $\Sigma \subseteq \mathbf{R}^{n}$ be a closed Jordan surface with boundary $\Gamma$ and let $\Sigma_{o}=\Sigma \backslash \Gamma$. Suppose that $f: \mathbf{U} \rightarrow \Sigma_{o}$ is a harmonic homeomorphism of the unit disk onto $\Sigma_{o}$ which may be represented in the form $f=\mathrm{P}[\phi]$, where the function $\phi$ is as in Theorem 2.2. We have: 
(1) If $\Gamma$ does not contain any segment, then $f$ has a continuous extension up to the boundary.

(2) If $\theta_{0} \in[0,2 \pi]$ is a point of discontinuity of $\phi$, then there exist

$$
A_{0}=\lim _{t \uparrow \theta_{0}} \phi(t), \quad B_{0}=\lim _{t \downarrow \theta_{0}} \phi(t)
$$

and

$$
C_{\mathbf{U}}\left(f, e^{i \theta_{0}}\right)=\left[A_{0}, B_{0}\right] \subseteq \Gamma .
$$

Proof. Take $\theta_{0} \in[0,2 \pi]$. There exist the left and the right boundary values of $\phi$ at $\theta_{0}$. Let $\lim _{\theta \uparrow \theta_{0}} f\left(e^{i \theta}\right)=A_{0}$ and $\lim _{\theta \downarrow \theta_{0}} f\left(e^{i \theta}\right)=B_{0}$.

For $R \geq 0$ and for $-1 \leq \lambda \leq 1$, let

$$
z_{R}=e^{i \theta_{0}}\left(1-R e^{-i \frac{\lambda \pi}{2}}\right) .
$$

Then $z_{R} \rightarrow e^{i \theta_{0}}$ as $R \rightarrow 0$ and the angle between the half line $\Gamma_{R}=\left\{z_{R}: 0 \leq R<\infty\right\}$ at $R=0$ and the point $e^{i \theta_{0}}$ is equal to $-\lambda \pi / 2$. In view of Proposition 2.3 we have

$$
\lim _{R \rightarrow 0} f\left(z_{R}\right)=\frac{1}{2}(1-\lambda) A_{0}+\frac{1}{2}(1+\lambda) B_{0} .
$$

It follows that $\left[A_{0}, B_{0}\right] \subseteq C_{\mathbf{U}}\left(f, e^{i \theta_{0}}\right)$. Since $f: \mathbf{U} \rightarrow \Sigma$ is a homeomorphism, it follows that $C_{\mathbf{U}}\left(f, e^{i \theta_{0}}\right) \subseteq \Gamma$. Therefore $\left[A_{0}, B_{0}\right] \subseteq \Gamma$.

If $\Gamma$ does not contain any segment then $A_{0}=B_{0}$, i.e., $\phi$ is continuous at $e^{i \theta}$. This proves the item (1).

In order to show (2), suppose that $\left[A_{0}, B_{0}\right] \nsubseteq C_{\mathbf{U}}\left(f, e^{i \theta_{0}}\right)$. Then there exists $\omega_{0} \in C_{\mathbf{U}}\left(f, e^{i \theta_{0}}\right) \backslash\left[A_{0}, B_{0}\right]$. We may assume that $\omega_{0}, A_{0}, B_{0}$ lie in the boundary $\Gamma$ in the positive direction and let $C$ be the arc in $\Gamma$ from $\omega_{0}$ to $B_{0}$. By Proposition 2.3 we could choose a Jordan arc $l$ in $\mathbf{U}$ such that the endpoints of $\bar{l}$ are $e^{i \theta_{1}}$ and $e^{i \theta_{2}}$ and such that $\left.f\right|_{l}$ has a continuous extension to $\bar{l}$ and $f\left(e^{i \theta_{1}}\right) \in \Gamma \backslash C, f\left(e^{i \theta_{2}}\right)$ lies in the interior of the arc from $\omega_{0}$ to $A_{0}$. Let $D$ be the Jordan domain bounded by $l$ and the boundary of $U$ which contains $e^{i \theta_{0}}$ on the boundary. By Proposition 2.3 and the definition of $C_{\mathbf{U}}\left(f, e^{i \theta_{0}}\right)$, there exist $\zeta_{n}$ and $z_{n}$ in $D$ such that $\zeta_{n} \rightarrow e^{i \theta_{0}}, z_{n} \rightarrow e^{i \theta_{0}}, f\left(\zeta_{n}\right) \rightarrow$ a point $\omega_{1}$ in $\left[A_{0}, B_{0}\right], f\left(z_{n}\right) \rightarrow \omega_{0}$. Then both $\omega_{0}$ and $\omega_{1}$ are on the boundary of $f(D)$, which is absurd. Thus $C_{\mathbf{U}}\left(f, e^{i \theta_{0}}\right) \in\left[A_{0}, B_{0}\right]$.

The first part of the previous theorem may be considered as Carathéodory theorem for closed Jordan harmonic surfaces. This generalizes one of the main results in the paper of Hengartner and Schober in [9], where the authors prove the same theorem but only for Jordan domains in the plane.

Define $\mathbf{T}_{r}=r \mathbf{T}$.

Lemma 2.5. Assume $\Sigma \subset \mathbf{R}^{n}$ is a closed Jordan surface spanned by a curve $\Gamma$ and suppose that $f: \mathbf{U} \rightarrow \Sigma_{o}=\Sigma \backslash \Gamma$ is a harmonic homeomorphism of the unit disk onto $\Sigma_{o}$. Let $\Gamma_{r}=f\left(\mathbf{T}_{r}\right), 0<r<1$ be the family of curves on the surface $\Sigma$. Then $\left|\Gamma_{r}\right|$ is increasing in $r$ and

$$
\left|\Gamma_{r}\right| \leq|\Gamma|
$$

(if a curve $\Gamma$ is not rectifiable, then we consider $|\Gamma|=\infty$ ).

In the proof we follow the outline of proof of Proposition 2.1 in [15].

Proof. We will assume that $\Gamma$ is rectifiable. There exists $\phi$ such that $f=\mathrm{P}[\phi]$ (Theorem 2.2). By (1), using integration by parts, it follows that $\partial_{\theta} f$ equals the 
Poisson-Stieltjes integral of $\phi$ (which we denote by PS $[\phi]$ ):

$$
\begin{aligned}
\partial_{\theta} f\left(r e^{i \theta}\right) & =\int_{0}^{2 \pi} \partial_{\theta} P(r, \theta-t) f(t) d t=-\int_{0}^{2 \pi} \partial_{t} P(r, \theta-t) f(t) d t \\
& =-\left.P(r, \theta-t) f(t)\right|_{t=0} ^{2 \pi}+\int_{0}^{2 \pi} P(r, \theta-t) d \phi(t)=\int_{0}^{2 \pi} P(r, \theta-t) d \phi(t) \\
& =\operatorname{PS}[\phi] .
\end{aligned}
$$

Denote by $T_{\phi}(t)=V_{0}^{t}(\phi)$ the total variation of $\phi$ on $[0, t](0 \leq t \leq 2 \pi)$ and define $V(\phi):=V_{0}^{2 \pi}(\phi)$. We have that

$$
\left|\partial_{\theta} f\left(r e^{i \theta}\right)\right| \leq \int_{0}^{2 \pi} P(r, \theta-t) d T_{\phi}(t) .
$$

Since $\int_{0}^{2 \pi} P(r, \theta-t) d \theta=1$, an application of Fubini's theorem yields

$$
\begin{aligned}
\left|\Gamma_{r}\right| & =\int_{0}^{2 \pi}\left|\partial_{\theta} f\left(r e^{i \theta}\right)\right| d \theta=\int_{0}^{2 \pi} d \theta\left|\int_{0}^{2 \pi} P(r, \theta-t) d \phi(t)\right| \\
& \leq \int_{0}^{2 \pi} d \theta \int_{0}^{2 \pi} P(r, \theta-t) d T_{\phi}(t)=\int_{0}^{2 \pi}\left(\int_{0}^{2 \pi} P(r, \theta-t) d \theta\right) d T_{\phi}(t) \\
& \leq \int_{0}^{2 \pi} d T_{\phi}(t) \leq V(\phi) \leq|\Gamma| .
\end{aligned}
$$

Actually, it is easy to check that $V(\phi)=|\Gamma|$. But $|\Gamma| \neq \int_{0}^{2 \pi}\left|\phi^{\prime}\right| d t$ in general since $\phi$ need not be absolutely continuous.

Since $\partial_{t} f$ is also harmonic, it follows that $\left|\partial_{t} f\right|$ is subharmonic and thus $\left|\Gamma_{r}\right|$ is increasing in $r$.

Lemma 2.6. Let $\Sigma_{o} \subseteq \mathbf{R}^{n}$ be an open Jordan surface bounded by a curve $\Gamma$ and let $f: \mathbf{U} \rightarrow \Sigma_{o}$ be a homeomorphism onto $\Sigma_{o}$. Suppose that $f$ has a continuous extension on $\mathbf{T}$, except for at most a countable set of points $\left\{a_{k}: k=1,2, \ldots\right\}$, where the cluster set $C_{\mathbf{U}}\left(f, a_{k}\right)$ is a segment. Further, suppose that the curves $\Gamma_{r}, 0<r<1$ defined by $f\left(r e^{i t}\right), 0 \leq t \leq 2 \pi$, are rectifiable. Then

$$
\limsup _{r \rightarrow 1}\left|\Gamma_{r}\right| \geq|\Gamma|,
$$

where again if $\Gamma$ is not rectifiable, then we consider $|\Gamma|=\infty$.

Proof. Let $d(x, y)=|x-y|$ be the distance between points $x$ and $y$ in $\mathbf{R}^{n}$ and

$$
E=\bigcup_{k \geq 1} C_{\mathbf{U}}\left(f, a_{k}\right) .
$$

Fix $\varepsilon>0$ and suppose that $\Gamma$ is rectifiable (if not, the proof is similar). There exist points $\omega_{0}, \omega_{1}, \ldots, \omega_{n} \in \Gamma$ such that

$$
\sum_{j=0}^{n} d\left(\omega_{j}, \omega_{j+1}\right)>|\Gamma|-\varepsilon / 2,
$$

where we set $\omega_{n+1}=\omega_{0}$. We may suppose w.l.g. that these points do not lie in $E$.

Since $f$ has a continuous extension onto the boundary of $\Sigma$ without segments, we can find points $\zeta_{j} \in \mathbf{T}$ such that $f\left(\zeta_{j}\right)=\omega_{j}$ for all $j=0,1, \ldots, n$. Let $\omega_{j}^{\prime}=f\left(r \zeta_{j}\right) \in$ 
$\Gamma_{r}$. The distance between $r \zeta_{j}$ and $\zeta_{j}$ is $1-r$ for each $j$. Since $n$ is fixed, there exists $r$ close enough to 1 such that

$$
s_{n}:=\sum_{j=0}^{n} d\left(\omega_{j}^{\prime}, \omega_{j}\right)<\varepsilon / 4 .
$$

Using the triangle inequality

$$
d\left(\omega_{j}, \omega_{j+1}\right) \leq d\left(\omega_{j}, \omega_{j}^{\prime}\right)+d\left(\omega_{j}^{\prime}, \omega_{j+1}^{\prime}\right)+d\left(\omega_{j+1}^{\prime}, \omega_{j+1}\right),
$$

we get

$$
\left|\Gamma_{r}\right| \geq \sum_{j=0}^{n} d\left(\omega_{j}^{\prime}, \omega_{j+1}^{\prime}\right) \geq \sum_{j=0}^{n} d\left(\omega_{j}, \omega_{j+1}\right)-2 s_{n}>|\Gamma|-\varepsilon .
$$

Since we can choose for $\varepsilon$ an arbitrary positive number, it follows $\lim _{\sup } \operatorname{sul}_{r \rightarrow 1}\left|\Gamma_{r}\right| \geq$ $|\Gamma|$.

The Smirnov theorem for holomorphic functions between planar domains can be generalized to the proper holomorphic mappings between the unit disk and holomorphic surfaces spanning on a rectifiable Jordan contour in $\mathbf{C}^{n}$. For this result see the paper of Globevnik and Stout [8]. This theorem can be generalized as well to harmonic $K$-quasiconformal mappings and $\left(K, K^{\prime}\right)$-quasiconformal harmonic mappings (see [12] and [19]). The following version of the Smirnov theorem holds for harmonic homeomorphisms (which are not quasiconformal in general) and in some sense it is optimal.

Theorem 2.7. Let $\Sigma_{o} \subseteq \mathbf{R}^{n}$ be an open Jordan surface spanned by a Jordan curve $\Gamma$ and let $f: \mathbf{U} \stackrel{\text { onto }}{\longrightarrow} \Sigma_{o}$ be a harmonic homeomorphism. Suppose that $\Gamma_{r}, 0<$ $r<1$, are the curves defined by $f\left(r e^{i t}\right), 0 \leq t \leq 2 \pi$. Then $\partial_{t} f \in h^{1}(\mathbf{U})$ if and only if $\Gamma$ is rectifiable. In this settings, $\left|\Gamma_{r}\right| \rightarrow|\Gamma|$ as $r \rightarrow 1$.

Proof. If $\Gamma$ is rectifiable, according to Lemma 2.5 we have $\left|\Gamma_{r}\right| \leq|\Gamma|$ what means

$$
\int_{0}^{2 \pi}\left|\partial_{t} f\left(r e^{i t}\right)\right| d t \leq|\Gamma|<\infty
$$

Thus $\partial_{t} f \in h^{1}(\mathbf{U})$.

On the other hand, if $\partial_{t} f \in h^{1}(\mathbf{U})$, then by Theorem 1.1 in [6] there exists $\phi$ which is of bounded variation and $\partial_{t} f=\operatorname{PS}[\phi]$, the Poisson-Stieltjes integral of $\phi$. Let $u=\mathrm{P}[\phi]$. Then $\partial_{t} u=\partial_{t} f$. Since the harmonic conjugate of $\partial_{t} u=\partial_{t} f$ is unique, we see that $r \partial_{r} u=r \partial_{r} f$. Thus we have $\partial_{t} u=\partial_{t} f$ and $\partial_{r} u=\partial_{r} f$. Therefore there exists a constant $c$ satisfying $f=u+c=\mathrm{P}[\phi+c]$. Then by Theorem $2.4, \psi:=\phi+c$ maps $[0,2 \pi]$ into $\Gamma$ and the assumption of Lemma 2.6 is satisfied, and by this lemma and Lemma 2.5, $|\Gamma|$ is finite.

Since we have a harmonic parametrization, $\left|\Gamma_{r}\right|$ is an increasing sequence. It follows $\lim _{r \rightarrow 1}\left|\Gamma_{r}\right| \leq|\Gamma|$ (by Lemma 2.5). We have the reverse inequality by Lemma 2.6. Thus $\lim _{r \rightarrow 1}\left|\Gamma_{r}\right|=|\Gamma|$.

Note that we have proved the next

Corollary 2.8. Let $\Sigma_{o} \subseteq \mathbf{R}^{n}$ be an open Jordan surface spanned by a Jordan curve $\Gamma$ and let $f: \mathbf{U} \stackrel{\text { onto }}{\longrightarrow} \Sigma_{o}$ be a harmonic homeomorphism. The following three conditions are equivalent to each other:

(1) there exists $\phi \in \mathrm{BV}[0,2 \pi]$ such that $\phi(0)=\phi(2 \pi)$ and $f=\mathrm{P}[\phi]$; 
(2) $\partial_{t} f \in h^{1}(\mathbf{U})$;

(3) $\Gamma$ is rectifiable.

Moreover, in this case $|\Gamma|=V(\phi)$.

In the settings of the previous corollary, in general, the parametrization for $\Gamma$ which is induced by $f$ is not always absolutely continuous (or even continuous). However if $n=2$ and $f$ is conformal, then $f$ induces on $\Gamma$ an absolutely continuous parametrization (this is the Smirnov theorem). Thus there is difference between harmonic diffeomorphisms and conformal diffeomorphism concerning the property of absolute continuity. See Proposition 2.1 in [3] and also [15].

\section{Some classical inequalities for harmonic surfaces-revisited}

Our first aim in this section is to establish the classical isoperimetric inequality for harmonic surfaces with rectifiable boundary (without any further smoothness assumption on the boundary). Some of results which we prove here may be known to the experts. Since we did not find broadly known references, we include their proofs for the convenience of readers.

3.1. Gaussian curvature of a smooth surface. Let $\Omega$ be a simply-connected domain in the plane. The first fundamental form of a (regular) surface $\Sigma \subseteq \mathbf{R}^{n}$ parametrized by a smooth mapping $\tau(z)=\left(\tau_{1}(z), \tau_{2}(z), \ldots, \tau_{n}(z)\right): \Omega \rightarrow \Sigma($ where $z$ $=x+i y)$ is given by

$$
d s^{2}=E d x^{2}+2 F d x d y+G d y^{2},
$$

here $E=g_{11}=\left|\tau_{x}\right|^{2}, F=g_{12}=\left\langle\tau_{x}, \tau_{y}\right\rangle$ and $G=g_{22}=\left|\tau_{y}\right|^{2}$ satisfy $E>0, F>0$ and $E G-F^{2}>0$ everywhere on $\Omega$.

The Gaussian curvature $K(x, y)$ of $\Sigma$ is usually expressed as a function of the first and second fundamental forms. However, for a surface which is not embedded in $\mathbf{R}^{3}$ the second fundamental form is not defined because it depends on the Gauss normal, which is not defined in a usual way in $\mathbf{R}^{n}$ when $n \geq 4$. The Brioschi formula for the Gaussian curvature gives us an opportunity to express it by

$$
K(x, y)=\frac{\left|\begin{array}{ccc}
-\frac{1}{2} E_{y y}+F_{x y}-\frac{1}{2} G_{x x} & \frac{1}{2} E_{x} & F_{x}-\frac{1}{2} E y \\
F_{y}-\frac{1}{2} G_{x} & E & F \\
\frac{1}{2} G_{y} & F & G
\end{array}\right|-\left|\begin{array}{ccc}
0 & \frac{1}{2} E_{y} & \frac{1}{2} G_{x} \\
\frac{1}{2} E_{y} & E & F \\
\frac{1}{2} G_{x} & F & G
\end{array}\right|}{\left(E G-F^{2}\right)^{2}} .
$$

This is indeed an alternative formulation of the fundamental Gauss Theorem Egregium and consequently the Gaussian curvature does not depend whether a surface is embedded in $\mathbf{R}^{3}$ or in some other Riemannian manifold.

For three vectors $a=\left(a_{1}, a_{2}, \ldots, a_{n}\right), b=\left(b_{1}, b_{2}, \ldots, b_{n}\right)$ and $c=\left(c_{1}, c_{2}, \ldots, c_{n}\right)$ we define the matrix

$$
[a, b, c]:=\left(\begin{array}{cccc}
a_{1} & a_{2} & \ldots & a_{n} \\
b_{1} & b_{2} & \ldots & b_{n} \\
c_{1} & c_{2} & \ldots & c_{n}
\end{array}\right)
$$

Lemma 3.1. Let $\Sigma$ be a surface in $\mathbf{R}^{n}$ with a parametrization $\tau=\tau(x, y)=$ $\left(\tau_{1}, \ldots, \tau_{n}\right)$ which is enough smooth. The Gaussian curvature can be expressed as

$$
K(x, y)=\frac{\operatorname{det}\left(\left[\tau_{x x}, \tau_{x}, \tau_{y}\right] \times\left[\tau_{y y}, \tau_{x}, \tau_{y}\right]^{t}\right)-\operatorname{det}\left(\left[\tau_{x y}, \tau_{x}, \tau_{y}\right] \times\left[\tau_{x y}, \tau_{x}, \tau_{y}\right]^{t}\right)}{\left(\left|\tau_{x}\right|^{2}\left|\tau_{y}\right|^{2}-\left\langle\tau_{x}, \tau_{y}\right\rangle^{2}\right)^{2}} .
$$


Remark 3.2. In standard expressions for Gaussian curvature, it appears the third derivatives of the parametrization. In formula (3) we have only the first and the second derivatives which is intrigue, but the proof depends on the third derivatives of $\tau$ as well and thus we should assume that the regularity of $\tau$ is something more than the membership on the class $C^{2}$.

Proof. We have the equalities

$$
\begin{aligned}
& E_{y}=2\left\langle\tau_{x y}, \tau_{x}\right\rangle, \quad E_{y y}=2\left\langle\tau_{x y y}, \tau_{x}\right\rangle+2\left|\tau_{x y}\right|^{2}, \\
& F_{x}=\left\langle\tau_{x x}, \tau_{y}\right\rangle+\left\langle\tau_{x}, \tau_{x y}\right\rangle, \quad F_{x y}=\left\langle\tau_{x x y}, \tau_{y}\right\rangle+\left\langle\tau_{x x}, \tau_{y y}\right\rangle+\left|\tau_{x y}\right|^{2}+\left\langle\tau_{x}, \tau_{x y y}\right\rangle, \\
& G_{x}=2\left\langle\tau_{x y}, \tau_{y}\right\rangle, \quad G_{x x}=2\left\langle\tau_{x x y}, \tau_{y}\right\rangle+2\left|\tau_{x y}\right|^{2},
\end{aligned}
$$

and

$$
-\frac{1}{2} E_{y y}+F_{x y}-\frac{1}{2} G_{x x}=\left\langle\tau_{x x}, \tau_{y y}\right\rangle-\left|\tau_{x y}\right|^{2}
$$

Thus

$$
\begin{aligned}
& \operatorname{det}\left(\left[\tau_{x y}, \tau_{x}, \tau_{y}\right] \times\left[\tau_{x y}, \tau_{x}, \tau_{y}\right]^{t}\right) \\
& =\left|\begin{array}{ccc}
\left|\tau_{x y}\right|^{2} & \frac{1}{2} E_{y} & \frac{1}{2} G_{x} \\
\frac{1}{2} E_{y} & E & F \\
\frac{1}{2} G_{x} & F & G
\end{array}\right|=\left|\begin{array}{ccc}
\left|\tau_{x y}\right|^{2} & 0 & 0 \\
\frac{1}{2} E_{y} & E & F \\
\frac{1}{2} G_{x} & F & G
\end{array}\right|+\left|\begin{array}{ccc}
0 & \frac{1}{2} E_{y} & \frac{1}{2} G_{x} \\
\frac{1}{2} E_{y} & E & F \\
\frac{1}{2} G_{x} & F & G
\end{array}\right|
\end{aligned}
$$

and

$$
\begin{aligned}
& \operatorname{det}\left(\left[\tau_{x x}, \tau_{x}, \tau_{y}\right] \times\left[\tau_{y y}, \tau_{x}, \tau_{y}\right]^{t}\right) \\
& =\left|\begin{array}{ccc}
\left|\tau_{x y}\right|^{2}-\frac{1}{2} E_{y y}+F_{x y}-\frac{1}{2} G_{x x} & \frac{1}{2} E_{x} & F_{x}-\frac{1}{2} E_{y} \\
F_{y}-\frac{1}{2} G_{x} & E & F \\
\frac{1}{2} G_{y} & F & G
\end{array}\right| \\
& =\left|\begin{array}{ccc}
\left|\tau_{x y}\right|^{2} & 0 & 0 \\
F_{y}-\frac{1}{2} G_{x} & E & F \\
\frac{1}{2} G_{y} & F & G
\end{array}\right|+\left|\begin{array}{ccc}
-\frac{1}{2} E_{y y}+F_{x y}-\frac{1}{2} G_{x x} & \frac{1}{2} E_{x} & F_{x}-\frac{1}{2} E_{y} \\
F_{y}-\frac{1}{2} G_{x} & E & F \\
\frac{1}{2} G_{y} & F & G
\end{array}\right| .
\end{aligned}
$$

The equality of the lemma now follows from Brioschi formula for Gaussian curvature.

\subsection{The isoperimetric inequality for harmonic surfaces.}

Theorem 3.3. If $\Sigma$ is a simply-connected harmonic surface which allows a regular harmonic parametrization $\tau$, then the Gaussian curvature of $\Sigma$ is nonpositive.

Proof. Let $\Sigma$ be a simply-connected harmonic surface with a regular harmonic parametrization $\tau$, that is, let $\Delta \tau=(0,0, \ldots, 0)$. Since $\tau_{y y}=-\tau_{x x}$, we obtain

$$
\begin{aligned}
& \operatorname{det}\left(\left[\tau_{x x}, \tau_{x}, \tau_{y}\right] \times\left[\tau_{y y}, \tau_{x}, \tau_{y}\right]^{t}\right)-\operatorname{det}\left(\left[\tau_{x y}, \tau_{x}, \tau_{y}\right] \times\left[\tau_{x y}, \tau_{x}, \tau_{y}\right]^{t}\right) \\
& =-\operatorname{det}\left(\left[\tau_{x x}, \tau_{x}, \tau_{y}\right] \times\left[\tau_{x x}, \tau_{x}, \tau_{y}\right]^{t}\right)-\operatorname{det}\left(\left[\tau_{x y}, \tau_{x}, \tau_{y}\right] \times\left[\tau_{x y}, \tau_{x}, \tau_{y}\right]^{t}\right) \leq 0,
\end{aligned}
$$

because the corresponding matrices are symmetric. The previous lemma implies that the Gaussian curvature of $\Sigma$ is non-positive.

Since the Gaussian curvature is an intrinsic invariant of a surface, from Theorem 3.3 we deduce the following result.

Theorem 3.4. (Isoperimetric inequality for harmonic surfaces) If $\Sigma \subseteq \mathbf{R}^{n}$ is a harmonic Jordan surface with the rectifiable boundary $\Gamma$, then we have the classical 
isoperimetric inequality

$$
4 \pi|\Sigma| \leq|\Gamma|^{2} .
$$

Proof. Let $\Sigma_{o}=\Sigma \backslash \Gamma$ and let $\tau: \mathbf{U} \stackrel{\text { onto }}{\longrightarrow} \Sigma_{o}$ be a harmonic parametrization of $\Sigma$. Since $\tau$ is not necessarily regular, as in [22], we add two extra dimensions to the target space and perturb the surface $\Sigma$ in $\mathbf{R}^{n+2}$. More precisely for $\varepsilon>0$ and $0<r<1$ let us consider the harmonic homeomorphism $\tau_{r}^{\varepsilon}(z)=(\tau(r z), \varepsilon z) \in$ $\mathbf{R}^{n+2}(z \in \mathbf{U})$, which is a regular harmonic parametrization of the simply-connected harmonic surface $\Sigma_{r}^{\varepsilon}=\tau_{r}^{\varepsilon}(\mathbf{U}) \subseteq \mathbf{R}^{n+2}$ with smooth boundary. Since (by the previous lemma) the Gaussian curvature of $\Sigma_{r}^{\varepsilon}$ is non-positive, applying the classical result (Theorem 4.1 in [18]), we obtain

$$
4 \pi\left|\Sigma_{r}^{\varepsilon}\right| \leq\left|\Gamma_{r}^{\varepsilon}\right|^{2}
$$

Letting first $\varepsilon \rightarrow 0$ and then $r \rightarrow 1$, by Theorem 2.7, we obtain (4).

We offer another proof of Theorem 3.4 by using the result of Beeson in [2] and Theorem 2.7. Since $\tau_{r}^{\varepsilon}$ converges to $\tau$ and $\left|\Gamma_{r}^{\varepsilon}\right|$ converges to $|\Gamma|$, it follows that $\left|\Sigma_{r}^{\varepsilon}\right|$ converges to $|\Sigma|$, and in view of (5) the inequality (4) follows immediately.

Remark 3.5. Theorem 3.4 can be considered as a variation of theorem of Shiffman [22]. Namely, in order to prove the isoperimetric inequality for harmonic surfaces $\Sigma$, Shiffman used the assumption that the harmonic parametrization $\tau$ is a homeomorphism with $\left.\tau\right|_{\mathbf{T}} \in \mathrm{BV}$. Our proof (see Theorem 2.4) shows that the condition $\left.\tau\right|_{\mathbf{T}} \in \mathrm{BV}$ is superfluous, if we assume (a topological condition) that $\Sigma$ is a Jordan surface with rectifiable boundary. In the famous Courant book [5] (see the proof of [5, Theorem 3.7]), which has been published some years after the paper of Shiffman it is proved for surfaces in euclidean 3-space (the case $n=3$ ) the following inequality holds

$$
4|\Sigma| \leq|\Gamma|^{2}
$$

under the condition $\Sigma=\tau(\mathbf{U})$, where $\tau$ is a harmonic parametrization with absolutely continuous boundary data.

Roughly speaking the isoperimetric inequality holds on sufficiently regular abstract surfaces (with the Riemannian metric) if and only if the Gaussian curvature is non-positive (cf. $[4,10,18]$ ) (This is a theorem of Beckenbach and Radó).

It is readable the corresponding version of Theorem 3.4 holds for Jordan minimal surfaces (and we expect that this particular case can be proved without Theorem 2.4). It is worth to notice the following important fact. For a minimal surface $\Sigma$ over a domain in the complex plane, every isothermal parametrization is a harmonic parametrization and it coincides with Enneper-Weierstrass parametrization of the minimal surface. Recall that Enneper-Weierstrass parameterization

$$
\tau(z)=\left(p_{1}(z), p_{2}(z), \ldots, p_{n}(z)\right), \quad z \in \mathbf{U},
$$

of a simply-connected minimal surface $\Sigma$ has harmonic coordinates $p_{j}(z), j=$ $1,2, \ldots, n$, such that $p_{j}(z)=\Re\left(a_{j}(z)\right)$, where $a_{j}, j=1,2, \ldots, n$, are analytic functions on the unit disk satisfying the equation $\sum_{j=1}^{n} a_{j}^{\prime}(z)^{2}=0$. It is clear that the Theorem 3.4 clarifies relations between some results discussed above and we expect that it will have further applications.

3.3. Riesz-Zygmund inequality. The following is a classical inequality of Riesz and Zygmund [24, Ch. IV, (6.28)]. 
Proposition 3.6. (Riesz-Zygmund inequality) If $f \in h^{1}$ is a harmonic function and $\partial_{t} f \in h^{1}$ then

$$
\int_{-1}^{1}\left|\partial_{r} f\left(r e^{i s}\right)\right| d r \leq \frac{1}{2}\left\|\partial_{t} f\right\|_{1}
$$

The constant $1 / 2$ is the best possible.

As a corollary we have the next inequality.

Corollary 3.7. Assume that $f$ is a harmonic diffeomorphism from the unit disc $\mathrm{U}$ onto a Jordan domain $\Omega$ with the rectifiable boundary $\Gamma$ and let $d$ be an arbitrary diameter of $\mathbf{U}$. Then, if by $|\cdot|$ we denote the corresponding length, we have

$$
|f(d)| \leq \frac{1}{2}|\Gamma|
$$

Now we prove the following extension of Proposition 3.6.

Theorem 3.8. (Riesz-Zygmund inequality for harmonic surfaces) Assume $\Sigma \subseteq$ $\mathbf{R}^{n}$ is a harmonic Jordan surface which is spanned by a rectifiable curve $\Gamma$ and parametrized by harmonic coordinates $\tau$. Then for every $s \in[0,2 \pi]$ we have

$$
l(s):=\int_{-1}^{1}\left|\partial_{r} \tau\left(r e^{i s}\right)\right| d t \leq \frac{1}{2}|\Gamma| .
$$

In other words, the length of the image of an arbitrary diameter $d$ of the unit disk under a harmonic parametrization $\tau$ is less than one half of the perimeter of the surface $\Sigma$.

Note that in the setting of this theorem $|\Gamma|=\left\|\partial_{t} f\right\|_{1}$, and if in addition $\tau$ is absolutely continuous on $\mathbf{T}$ then $|\Gamma|=\int_{0}^{2 \pi}\left|\partial_{t} \tau\left(e^{i t}\right)\right| d t$.

Proof. Assume that $\tau$ are harmonic coordinates. By Theorem 2.2, there exists $\phi \in \mathrm{BV}[0,2 \pi]$ such that $\phi(0)=\phi(2 \pi)$. Then as in the proof of Lemma 2.5, $\partial_{t} \tau$ is represented by $\partial_{t} \tau=\operatorname{PS}(\phi)$. Further, let $\tau=\left(\Re\left(a_{1}\right), \Re\left(a_{2}\right), \ldots, \Re\left(a_{n}\right)\right)$, where $a_{j}$, $j=1,2, \ldots, n$ are analytic function in the unit disk. Then

$$
\partial_{t} \tau+i r \partial_{r} \tau=\left(a_{1}^{\prime}, a_{2}^{\prime}, \ldots, a_{n}^{\prime}\right) \in \mathbf{C}^{n}
$$

and thus $r \partial_{r} \tau$ is the harmonic conjugate of $\partial_{t} \tau$. Define

$$
F(z)=(1+z) /(1-z), \quad Q(r, t)=\Im F\left(r e^{i t}\right)=2 r \sin t|1-z|^{-2}
$$

and

$$
K(r, t)=r^{-1} Q(r, t), \quad K_{+}(r, t)=|K(r, t)|=2|\sin t||1-z|^{-2}
$$

(where $z=r e^{i t}$ ). It is known that

$$
r \partial_{r} \tau\left(r e^{i s}\right)=\frac{1}{2 \pi} \int_{-\pi}^{\pi} Q(r, t) d \phi(s-t)
$$

Hence

$$
2 \pi\left|\partial_{r} \tau\left(r e^{i s}\right)\right| \leq \int_{-\pi}^{\pi} K_{+}(r, t)|d \phi(s-t)| d t
$$

Now we use that

$$
A(t)=\int_{-1}^{1} K_{+}(r, t) d r=\pi
$$


for $0<|t|<\pi$ (this is an elementary result). By Fubini's theorem, (8) and (9) we obtain

$$
\begin{aligned}
l(s) & =\int_{-1}^{1}\left|\partial_{r} \tau\left(r e^{i s}\right)\right| d r \leq \frac{1}{2 \pi} \int_{0}^{2 \pi}\left(\int_{-1}^{1} K_{+}(r, t) d r\right)|d \phi(s-t)| \\
& \leq \frac{1}{2 \pi} \int_{0}^{2 \pi} A(t)|d \phi(s-t)|=\frac{1}{2} \int_{0}^{2 \pi}|d \phi(s-t)|=\frac{1}{2} \int_{0}^{2 \pi}|d \phi(t)| .
\end{aligned}
$$

Let $\Sigma \subseteq \mathbf{R}^{n}$ be a regular surface. For two points $P, Q \in \Sigma$ we define the intrinsic distance as follows

$$
d_{I}(P, Q)=\inf _{c \in \mathfrak{C}}|c|,
$$

where $\mathfrak{C}$ is the set of all Jordan $\operatorname{arcs} c$ of $\Sigma$ with the length $|c|$ connecting $P$ and $Q$. It should be noted the following fact, for close enough points $P$ and $Q$ it exists a geodesic line $\gamma$ connecting $P$ and $Q$ such that $d_{I}(P, Q)=|\gamma|$. We define the (geodesic) diameter of $\Sigma$ as

$$
\operatorname{diam}(\Sigma)=\sup _{P, Q \in \Sigma} d_{I}(P, Q) .
$$

We can now deduce the following geometric application of Theorem 3.8.

Theorem 3.9. If $\Sigma \subseteq \mathbf{R}^{n}$ is an arbitrary harmonic Jordan surface with rectifiable boundary $\Gamma$, then

$$
\operatorname{diam}(\Sigma) \leq \frac{1}{2}|\Gamma|
$$

The constant $\frac{1}{2}$ is the best possible even for minimal surfaces lying over the unit disk.

The diameter of the harmonic surface in Figure 2 is bigger than the half of the length of its boundary and this surface shows that the assumption in the previous theorem that $\Sigma$ is a harmonic Jordan surface is essential.

Using an approach as in the proof of Theorem 3.8, we obtain

Theorem 3.10. If $f: \mathbf{U} \rightarrow \mathbf{R}^{n}$ is a harmonic function and $\partial_{t} f \in h^{1}$, then

$$
l(s):=\int_{-1}^{1}\left|\partial_{r} f\left(r e^{i s}\right)\right| d r \leq \frac{1}{2}\left\|\partial_{t} f\right\|_{1} .
$$

Theorem 3.11. Suppose that $\psi:[0,2 \pi] \rightarrow \mathbf{R}^{n}$ is of bounded variation and $f=\mathrm{P}[\phi]$. Then

$$
\int_{-1}^{1}\left|\partial_{r} f\left(r e^{i s}\right)\right| d r \leq \frac{1}{2} V(\phi)
$$

Proof. An application of Theorem 3.10 yields

$$
\int_{-1}^{1}\left|\partial_{r} f\left(r_{0} r e^{i s}\right)\right| d r \leq \frac{1}{2} L\left(r_{0}\right)
$$

where

$$
L\left(r_{0}\right)=\int_{0}^{2 \pi}\left|\partial_{t} f\left(r_{0} r e^{i t}\right)\right| d t
$$

for $0<r_{0}<1$. By Proposition 2.1 in [15], $L\left(r_{0}\right) \leq V(\phi)$ and therefore $l(s) \leq \frac{1}{2} V(\phi)$, if $r_{0} \rightarrow 1$.

Define $\mathbf{U}_{r}=\{z:|z|<r\}$. 
Theorem 3.12. Suppose that $\phi:[0,2 \pi] \rightarrow \mathbf{R}^{n}$ is of bounded variation, $f=\mathrm{P}[\phi]$ and $D=f(\mathbf{U})$. Then

(where $\left.V(\phi):=V_{0}^{2 \pi}(\phi)\right)$.

$$
\operatorname{diam}(D) \leq \frac{1}{2} V(\phi)
$$

Proof. For $0<r<1$, let $D_{r}=f\left(\mathbf{U}_{r}\right)$ and $C_{r}$ the curve defined by $f\left(r e^{i t}\right)$, $0 \leq t \leq 2 \pi$. For $0<r<1$, an application of Theorem 3.9 yields

$$
\operatorname{diam}\left(D_{r}\right) \leq \frac{1}{2}\left|C_{r}\right|
$$

Hence, if $r \rightarrow 1$, using the proof of Lemma 2.5 we conclude that $\operatorname{diam}(D) \leq \frac{1}{2} V(\phi)$.

Proof of Theorem 3.9. Since $\Sigma$ is a harmonic Jordan surface there is a regular harmonic parametrization $\tau: \mathbf{U} \rightarrow \Sigma$ of the surface $\Sigma$. We can suppose that $\tau$ is a regular parametrization, because if it is not the case we can perturb surface in $\mathbf{R}^{n+2}$ as in the proof of the isoperimetric inequality. Take $P, Q \in \Sigma \backslash \Gamma$ arbitrary. It suffices to show $d_{I}(P, Q) \leq \frac{1}{2}|\Gamma|$. There exists a conformal mapping $a$ of the unit disk $\mathbf{U}$ onto itself such that $\tau(a(-x))=P$ and $\tau(a(x))=Q, 0<x<1$. Take $v_{\delta}(z)=(\tau \circ a)(\delta z), x<\delta<1$. Then by Theorem 3.8 and relation (2) we have

$$
d_{I}(P, Q) \leq \int_{-1}^{1}\left|\partial_{r} v_{\delta}(r)\right| d r<\frac{1}{2} \int_{0}^{2 \pi}\left|\partial_{t} v_{\delta}\left(e^{i t}\right)\right| d t \leq \frac{1}{2}|\Gamma| .
$$

By $d_{I}(P, Q)<\frac{1}{2}|\Gamma|$ we obtain $(10)$.

Show that the constant $1 / 2$ is sharp. Assume, as we may that $n=3$. Let $d=\left[-e^{i t}, e^{i t}\right]$ be an arbitrary diameter of the unit disk and let

$$
\tau(x, y)=(x, y, m(x+y))
$$

where $m$ is a large constant. We can express the perimeter of the minimal surface $\tau$ by Elliptic integral of the second kind $E$, i.e.,

$$
|\Gamma|=2\left(E\left(\pi / 4,-2 m^{2}\right)+E\left((3 \pi) / 4,-2 m^{2}\right)\right) .
$$

The length of $\tau(d)$ is $2 \sqrt{1+m^{2}+m^{2} \sin 2 t}$. The maximal diameter is attained for $t=\pi / 4$ and is equal $2 \sqrt{1+2 m^{2}}$. Then

$$
\lim _{m \rightarrow \infty} \frac{2 \sqrt{1+2 m^{2}}}{2\left(E\left(\pi / 4,-2 m^{2}\right)+E\left(3 \pi / 4,-2 m^{2}\right)\right)}=\frac{1}{2},
$$

what proves that $1 / 2$ is an optimal constant for the inequality.

Acknowledgement. M. Mateljević was supported by Serbian Ministry of Science, project number 174032. D. Kalaj was supported by Ministry of Science of Montenegro. We are particularly grateful to an anonymous referee for very constructive numerous advices which improved this paper.

\section{References}

[1] Alexander, H.: Polynomial hulls and linear measure. - In: Complex analysis II, Proc. Univ. of Maryland, Lecture Notes in Math. 1275, Springer-Verlag, Berlin-Heidelberg-New York, 1987, $1-11$.

[2] Beeson, M.: On the area of harmonic surfaces. - Proc. Amer. Math. Soc. 69, 1978, $143-147$. 
[3] BožIn, V., M. MatelJević, and M. KneŽEvić: Quasiconformality of harmonic mappings between Jordan domains. - Filomat 24, 2010, 111-124.

[4] Chavel, I.: Riemmanian geometry: A modern introduction. 2nd edition. - Cambridge Univ. Press, Cambridge, 2006.

[5] Courant, R.: Dirichlet's principle, conformal mapping, and minimal surfaces. With an appendix by M. Schiffer. Reprint of the 1950 original. - Springer-Verlag, New York-Heidelberg, 1977.

[6] Duren, P.: Theory of $H^{p}$ spaces. - Academic Press, New York and London, 1970.

[7] Duren, P.: Harmonic mappings in the plane. - Cambridge Univ. Press, 2004.

[8] Globevnik, J., and E. L. Stout: Analytic discs with rectifiable simple closed curves as ends. - Ann. of Math. (2) 127, 1988, 389-401.

[9] Hengartner, W., and G. Schober: Harmonic mappings with given dilatation. - J. London Math. Soc. (2) 33, 1986, 473-483.

[10] Huber, A.: On the isoperimetric inequality on surfaces of variable Gaussian curvature. - Ann. of Math. (2) 60, 1954, 237-247.

[11] Jost, J.: Harmonic maps between surfaces. - Lecture Notes in Math. 1062, Springer-Verlag, Berlin, 1984.

[12] Kalaj, D., and M. Mateljević: $\left(K, K^{\prime}\right)$-quasiconformal harmonic mappings. - Potential Anal. 36, 2012, 117-135.

[13] Lewy, H.: On the non-vanishing of the Jacobian in certain in one-to-one mappings. - Bull. Amer. Math. Soc. 42, 1936, 689-692.

[14] Lozinski, S.: On subharmonic functions and their application to the theory of surfaces. - Izv. Akad. Nauk SSSR Ser. Mat. 8:4, 1944, 175-194.

[15] Mateljević, M.: Quasiconformality of harmonic mappings between Jordan domains 2. Filomat 26:3, 2012, 479-510.

[16] Mateljević, M., and M. Pavlović: Some inequalities of isoperimetric type concerning analytic and subharmonic functions. - Publ. Inst. Math. (Beograd) (N.S.) 50:64, 1991, 123-130.

[17] Natanson, I. P.: Theory of functions of a real variable. - Ungar, New York, 1955 and 1960.

[18] Osserman, R.: The isoperimetric inequality. - Bull. Amer. Math. Soc. 84, 1978, 1182-1238.

[19] Partyka, D. and K. SAKAn: On bi-Lipschitz type inequalities for quasiconformal harmonic mappings. - Ann. Acad. Sci. Fenn. Math. 32, 2007, 579-594.

[20] Pommerenke, Ch.: On analytic functions with cluster sets of finite linear measure. - Michigan Math. J. 34, 1987, 93-99.

[21] Scheeffer, L.: Allgemeine Untersuchungen über Rectification der Curven. - Acta Math. 5, $1885,49-82$.

[22] Shiffman, M.: On the isoperimetric inequality for saddle surfaces with singularities. - In: Studies and Essays Presented to R. Courant on his 60th Birthday, January 8, 1948, Interscience Publishers, New York, 1948, 383-394.

[23] Weil, A.: Sur les surfaces a courbure negative. - C. R. Acad. Sci. Paris 182, 1926, 1069-1071.

[24] Zygmund, A.: Trigonometric series. Volumes I and II. 2nd edition. - Cambridge Univ. Press, New York, 1959. 